\title{
Timing of radiotherapy and outcome in patients receiving adjuvant endocrine therapy
}

\author{
Per Karlsson, MD, \\ Department of Oncology, Sahlgrenska University Hospital, Gothenburg, Sweden. \\ per.karlsson@oncology.gu.se
}

\section{Bernard F. Cole, PhD,}

Department of Mathematics and Statistics College of Engineering and Mathematical Sciences, University of Vermont, Burlington, VT, USA and IBCSG Statistical Center, Department of Biostatistics and Computational Biology, Dana-Farber Cancer Institute, Boston, MA, USA. ccole@cems.uvm.edu

\section{Marco Colleoni, MD, Department of Medicine, Research Unit in Medical Senology, European Institute of Oncology, Milan, Italy. marco.colleoni@ieo.it}

Mario Roncadin, MD, Department of Radiotherapy. Centro di Riferimento Oncologico, Aviano, Italy.mroncadin@cro.it

Boon Chua, MD, PhD, Department of Radiation Oncology, Peter MacCallum Cancer Centre, Melbourne, Australia. Boon.Chua@petermac.org

Elizabeth Murray, MD, Department of Radiation Oncology. Groote Shuur Hospital and University of Cape Town, South Africa. Elizabeth.Murray@uct.ac.za

Karen N. Price, BS, IBCSG Statistical Center, Frontier Science and Technology Research Foundation, Boston, MA, USA. price@jimmy.harvard.edu

Monica Castiglione-Gertsch, MD, IBCSG Coordinating Center, Bern, Switzerland. monica.castiglione@bluewin.ch

Aron Goldhirsch, MD, and European Institute of Oncology, Milan, Italy and Oncology Institute of Southern Switzerland, Bellinzona, Switzerland aron.goldhirsch@ibcsg.org

\section{Günther Gruber, MD}

\footnotetext{
(C) 2010 Elsevier Inc. All rights reserved.

Correspondence prior to publication: Karen N. Price, Department of Biostatistics and Computational Biology, Dana-Farber Cancer Institute, 44 Binney Street, Boston, MA 02115, Telephone: 617632 2459; Fax: 617632 5444; price@ jimmy.harvard.edu. Address reprint requests to: Per Karlsson, M.D., Department of Oncology, University of Göteborg, Sahlgrenska University Hospital, S 41345 Gothenburg, Sweden Telephone: +46 31342 2246; Fax: +46 31 820114; per.karlsson@oncology.gu.se

Publisher's Disclaimer: This is a PDF file of an unedited manuscript that has been accepted for publication. As a service to our customers we are providing this early version of the manuscript. The manuscript will undergo copyediting, typesetting, and review of the resulting proof before it is published in its final citable form. Please note that during the production process errors may be discovered which could affect the content, and all legal disclaimers that apply to the journal pertain.

Prior presentation: Preliminary results were presented in a poster at the St. Gallen Breast Conference, St. Gallen, Switzerland, March 2009

Conflicts of Interest Notification: None of the authors have any conflicts of interest relative to the content of this paper.
} 
Institut fuer Radiotherapie, Klinik Hirslanden, Zürich, Switzerland guenther.gruber@hirslanden.ch

\section{for the International Breast Cancer Study Group}

\section{Abstract}

Purpose-To evaluate the association between time from breast-conserving surgery (BCS) to radiotherapy and clinical outcome among patients treated with adjuvant endocrine therapy.

Methods-Patient information was obtained from three International Breast Cancer Study Group trials. Analysis was restricted to 964 patients treated with BCS and adjuvant endocrine therapy. Patients were divided into two groups based on the median number of days between BCS and radiotherapy and into 4 groups based on the quartile of time between BCS and radiotherapy. Endpoints were time to local recurrence (TLR), disease-free survival (DFS) and overall survival (OS). Proportional hazards regression analysis was used to perform comparisons after adjustment for baseline factors.

Results-The median time between BCS and radiotherapy was 77 days. Radiotherapy timing was significantly associated with age, menopausal status, and estrogen receptor status. After adjustment for these factors no significant effect of radiotherapy delay of up to 20 weeks was found. Adjusted hazard ratios (HRs) (radiotherapy within 77 days vs. after 77 days) were 0.94 (95\% CI 0.47-1.87) for TLR, 1.05 (95\% CI 0.82-1.34) for DFS and 1.07 (95\% CI 0.77-1.49) for OS. For TLR the adjusted HRs for $\leq 48$ days, 49-77 days, and 78-112 days were 0.90 (CI 95\% $0.34-2.37$ ), 0.89 (95\% CI, $0.33-2.25$ ), and 0.89 (95\% CI, $0.33-2.41$ ), respectively relative to $\geq$ 113 days.

Conclusions-Radiotherapy delay of up to 20 weeks was significantly associated with baseline factors such as age, menopausal status, and estrogen-receptor status. After adjustment for these factors, timing of radiotherapy was not significantly associated with TLR, DFS, or OS.

\section{Keywords}

breast cancer; radiotherapy; radiotherapy timing; breast-conserving surgery; endocrine therapy

\section{Introduction}

Radiotherapy to the breast after breast-conserving surgery reduces the risk for local recurrence and improves breast cancer specific survival [1]. The optimal time between surgery and the start of radiotherapy is not known. Theoretically, the risk of recurrence is related to the density of clonogenic cells in the surgical bed. Therefore, a delay between surgery and the start of radiotherapy may increase the likelihood of tumor cell growth and development of radioresistance [2]. Several retrospective studies have yielded variable results [3-8]. Generally, in univariate analysis, an increased risk for local recurrence was observed with longer delay between surgery and the start of radiotherapy. However, in multivariate analysis, this effect was not observed. Two systematic reviews showed an increase in the risk of local recurrence with radiotherapy delay of longer than 8 weeks $[9,10]$. However, these reviews included all subtypes of breast cancer and a variety of study designs rendering interpretation of the results difficult. The risk of local recurrence in relation to radiotherapy delay may vary by breast cancer subtype and systemic treatment. An International Breast Cancer Study Group (IBCSG) study has shown that delaying radiotherapy until the completion of chemotherapy does not adversely affect treatment outcome [11]. There has been no study that examines the effect of radiotherapy delay on local recurrence in breast cancer exclusively in patients receiving endocrine therapy. The aim of the present study is to investigate the impact of delay from breast-conserving surgery 
(BCS) to the start of radiotherapy in patients treated with endocrine therapy in three IBCSG trials.

\section{Patients and Methods}

Patient information was obtained from 1,108 patients who had BCS and were randomized to selected treatment arms from IBCSG trials VII, VIII and IX. Of the 1,108 patients identified, 135 did not receive radiotherapy and an additional nine did not have a record of radiotherapy commencement dates, leaving 964 patients in the analyzed cohort.

Trial VII compared adjuvant tamoxifen alone vs. chemoendocrine treatments of tamoxifen with concurrent classical cyclophosphamide, methotrexate, and 5-fluorouricil (CMF) in postmenopausal patients with node-positive breast cancer. [12]. Trial VIII studied adjuvant ovarian function suppression with LH-RH analogue for two years vs. six courses of CMF vs. six courses of CMF followed by 18 months of ovarian function suppression in premenopausal patients with node-negative disease [13]. Trial IX compared adjuvant tamoxifen vs. 3 courses of CMF followed by tamoxifen in postmenopausal patients with node-negative disease [14]. The present study was restricted to the following treatment groups receiving adjuvant endocrine therapy: trial VII Arm A (tamoxifen for 5 years) $(\mathrm{n}=69)$, trial VIII Arm B (LH-RH analogue for two years) $(\mathrm{n}=173)$, trial IX Arm A (tamoxifen for 5 years) $(\mathrm{n}=374)$, and trial IX Arm B (CMFx3 followed by tamoxifen for 57 months) ( $n=370)$. Trial VII specified that radiotherapy was required for all patients who had BCS. Although trials VIII and IX did not mandate radiotherapy to the conserved breast, $88 \%$ of the patients who had BCS were treated with radiotherapy. In all three trials, patients who had BCS and radiotherapy and were randomized to receive endocrine therapy alone were required to begin radiotherapy within three months of randomization, and those randomized to receive CMF prior to tamoxifen in trial IX were to begin radiotherapy two weeks after the end of the last cycle of chemotherapy. For the present study, patients were divided into two groups based on the median number of days from surgery to commencement of radiotherapy. Kaplan-Meier survival curves were plotted for the two groups, and the logrank test was used to compare them. The endpoints were time to local recurrence, diseasefree survival (DFS) and overall survival (OS), measured from start of radiotherapy. This approach accounts for the fact that the time to an event must exceed the number of days between surgery and the start of radiotherapy. In addition, patients were divided into four groups based on the quartiles of days from surgery until start of radiotherapy. Proportional hazards regression was used to estimate hazard ratios (HRs) and 95\% confidence intervals (CIs) for the 2-group and 4-group analyses. Wald tests were used to evaluate heterogeneity among the HRs based on the 4-group analysis. Of the 964 patients in the analytic cohort, 715 had estrogen receptor (ER)-positive disease. All analyses were repeated for the ERpositive cohort.

\section{Results}

The median number of days from surgery to the start of radiotherapy was 77 days (mean 84 days, standard deviation 45 days, range 14 to 436 days). 409 patients ( $42 \%$ ) received radiotherapy more than 90 days following surgery. Table 1 shows the baseline characteristics of the two groups of patients who started radiotherapy within or after 77 days (median number of days) from surgery. Age, pre-menopausal status, and hormone receptor status were significantly associated with radiotherapy timing. However it should be noted that some of these differences may be due to the CMF-tamoxifen arm of Trial IX in which patients had radiotherapy after chemotherapy. 
The median follow-up was 10.5 years. The total numbers of events were 37 local recurrences, 271 disease-free survival events and 161 deaths. Adjusting for the differences in baseline characteristics the HR for starting radiotherapy within 77 days relative to after 77 days was 0.94 (95\% CI 0.47-1.87) for local recurrence, 1.05 (95\% CI 0.82-1.34) for DFS and 1.07 (95\% CI 0.77-1.49) for OS (Table 2). When patients were divided into quartiles according to surgery-to-radiotherapy interval the adjusted HRs were not significantly different (Table 3). The Kaplan-Meier curves for time to local recurrence, DFS and OS with time to event from the start of radiotherapy for the two-group analysis and for the four-group analysis are shown in Figure 1 and Figure 2 respectively. Kaplan-Meier analysis with time to event measured from day 77 after surgery gave similar results (data not shown). The analyses were repeated for the ER-positive cohort $(n=715)$ with similar results (Table 2B and Table 3B), and there were no significant interactions between RT timing and ERpositivity (data not shown).

The mean time from surgery to the start of endocrine therapy was 33 days in the group that started radiotherapy within 77 days and 81 days in the group that started radiotherapy after 77 days. Among the 841 patients (89\%) who began endocrine therapy prior to radiotherapy, the mean time from start of endocrine therapy to start of radiotherapy was 26 days in the group that started radiotherapy within 77 days and 48 days in the group that started radiotherapy after 77 days.

\section{Discussion}

After adjustment for differences in the baseline characteristics, radiotherapy timing was not significantly associated with time to local recurrence, DFS or OS in patients receiving adjuvant endocrine therapy for radiotherapy delay of up to about 20 weeks. Postoperative radiotherapy after breast conserving surgery reduces local failure rates substantially and is therefore well accepted [1], but the optimal time interval between surgery and start of irradiation is still controversial. A recent meta-analysis by Chen et al indicated an increasing relative risk of 1.11 per month of irradiation delay for local recurrence in so-called 'high quality' series, irrespective of whether chemotherapy was given or not [9], but there was no significant impact on distant metastasis or overall survival. This meta-analysis confirmed a previous systematic review [10], in which the 5-year local relapse rate was significantly higher in breast cancer patients starting adjuvant radiotherapy more than 8 weeks after surgery compared to patients treated within 8 weeks (odds ratio: 1.62; 95\% CI 1.21-2.16). However, these meta-analyses included patients with a variety of breast cancer subtypes and in whom the use of systemic therapy was inconsistent. Both of these factors may have an impact on the local recurrence rate. Only a few studies have evaluated the time interval of radiotherapy after surgery in the absence of chemotherapy. The more recent meta-analysis [9] identified only four studies $[3,5,15,16]$, all of them observational, but with an adequate description of the distribution of relevant prognostic factors, which were either well balanced between the comparison groups or appropriately adjusted for in the analyses. Among the individual studies, the largest by far [5] examined the impact of radiotherapy delay of $<6$ weeks, 6-8 weeks, 9-12 weeks and $\geq 13$ weeks on 1,962 patients who had BCS. Of the 1,962 patients, 677 received tamoxifen and no patients had chemotherapy. Twentythree patients had a radiotherapy delay of longer than 20 weeks. At a median follow-up was 71 months, the local recurrence rate was significantly associated with the omission of tamoxifen and high histologic grade of the tumors. The interval between BCS and radiotherapy was not significantly associated with local recurrence in a model incorporating tamoxifen use and histologic grade, which was in contrast to the overall finding of the metaanalysis, but consistent with our results. 
A more recent publication from the British Columbia Cancer Agency examined different time intervals between surgery and radiotherapy in early-stage breast cancer patients in the absence of chemotherapy [8]: The study yielded similar results as the present study for radiotherapy delay of up to 20 weeks However, radiotherapy delay of more than 20 weeks resulted in an inferior outcome. Patients who were not given endocrine therapy were separately analyzed and findings were similar as for the entire cohort, but there was no separate subgroup analysis for patients who were given endocrine therapy[8]. In a recent study from Florence [4] the risk of developing breast failure for patients without any systemic adjuvant treatment $(\mathrm{n}=1935)$ was inversely proportional to the interval between surgery and radiotherapy in univariate analysis, but timing of radiotherapy was not statistically significance in a multivariate analysis. Similar results were obtained in the treatment groups of radiotherapy/endocrine therapy $(n=1684)$ and radiotherapy/endocrine therapy/chemotherapy $(n=529)$. Radiotherapy timing was statistically significant in multivariate analysis only in the group of patients with radiotherapy and chemotherapy $(\mathrm{n}=672)$ (hazard ratio: 1.69; $\mathrm{p}=0.045)$ [4]. This paper also did not provide separate analyses for patients with radiotherapy and endocrine therapy. In a previous publication of our group we analyzed the impact of radiotherapy delay due to chemotherapy in IBCSG trials VI and VII [11]. The current analysis was undertaken as studies investigating radiotherapy timing in patients with endocrine therapy are rare. Similar to others [4], we could not find any impact of the time interval from surgery to radiotherapy on local control or other endpoints.

However, the present study has too few events to provide an accurate estimate of the effect of radiotherapy delay of over 20 weeks. In addition, a limitation of the present study is the insufficient power to reject an association between radiotherapy delay of shorter intervals and a small increase in the local recurrence risk. Nevertheless, and despite its retrospective design, the current study deals with patients treated in a randomized study with defined inclusion/exclusion criteria, uniform adjuvant therapy/-ies and rigid follow-up control, which is in contrast to most observational series available in the literature.

The question of the effect of sequential or concurrent use of endocrine treatment and radiotherapy on tumor control could not be addressed in the present study. In IBCSG Trials VII-IX, most patients started endocrine therapy within two days after randomization, except for those assigned to receive CMF first, and radiotherapy was administered concurrently with endocrine therapy.

A study of IBCSG Trials VI and VII showed that a delay of radiotherapy from BCS of up to 6.6 months (28 weeks), during which chemotherapy was given, did not adversely influence treatment outcome [11]. The present IBCSG study indicates that an interval of up to about 20 weeks between breast conserving surgery and radiotherapy does not increase the risk of local recurrence in a cohort of patients receiving standard adjuvant endocrine treatment.

\section{Acknowledgments}

We thank the patients, physicians, nurses, and data managers who participate in the International Breast Cancer Study Group trials. The IBCSG is funded in part by: Swiss Group for Clinical Cancer Research, Frontier Science and Technology Research Foundation, The Cancer Council Australia, Australian New Zealand Breast Cancer Trials Group (National Health Medical Research Council), National Cancer Institute (CA-75362), Swedish Cancer Society, Cancer Association of South Africa, Foundation for Clinical Cancer Research of Eastern Switzerland (OSKK).

\section{References}

1. Clarke M, Collins R, Darby S, et al. Effects of radiotherapy and of differences in the extent of surgery for early breast cancer on local recurrence and 15-year survival: an overview of the randomised trials. Lancet. 2005; 366:2087-2106. [PubMed: 16360786] 
2. Fletcher GH. Implications of the density of clonogenic infestation in radiotherapy. Int J Radiat Oncol Biol Phys. 1986; 12:1675-1680. [PubMed: 3531119]

3. Clarke DH, Le MG, Sarrazin D, et al. Analysis of local-regional relapses in patients with early breast cancers treated by excision and radiotherapy: experience of the Institut Gustave-Roussy. Int J Radiat Oncol Biol Phys. 1985; 11:137-145. [PubMed: 2981790]

4. Livi L, Borghesi S, Saieva C, et al. Radiotherapy timing in 4,820 patients with breast cancer: university of florence experience. Int J Radiat Oncol Biol Phys. 2009; 73:365-369. [PubMed: 18715726]

5. Froud PJ, Mates D, Jackson JS, et al. Effect of time interval between breast-conserving surgery and radiation therapy on ipsilateral breast recurrence. Int J Radiat Oncol Biol Phys. 2000; 46:363-372. [PubMed: 10661343]

6. Benk V, Joseph L, Fortin P, et al. Effect of delay in initiating radiotherapy for patients with early stage breast cancer. Clin Oncol (R Coll Radiol). 2004; 16:6-11. [PubMed: 14768749]

7. Hebert-Croteau N, Freeman CR, Latreille J, Brisson J. Delay in adjuvant radiation treatment and outcomes of breast cancer--a review. Breast Cancer Res Treat. 2002; 74:77-94. [PubMed: 12150455]

8. Olivotto IA, Lesperance ML, Truong PT, et al. Intervals longer than 20 weeks from breastconserving surgery to radiation therapy are associated with inferior outcome for women with earlystage breast cancer who are not receiving chemotherapy. J Clin Oncol. 2009; 27:16-23. [PubMed: 19018080]

9. Chen Z, King W, Pearcey R, et al. The relationship between waiting time for radiotherapy and clinical outcomes: a systematic review of the literature. Radiother Oncol. 2008; 87:3-16. [PubMed: 18160158]

10. Huang J, Barbera L, Brouwers M, et al. Does delay in starting treatment affect the outcomes of radiotherapy? A systematic review. J Clin Oncol. 2003; 21:555-563. [PubMed: 12560449]

11. Wallgren A, Bernier J, Gelber RD, et al. Timing of radiotherapy and chemotherapy following breast-conserving surgery for patients with node-positive breast cancer. Int J Radiat Oncol Biol Phys. 1996; 35:649-659. [PubMed: 8690630]

12. International Breast Cancer Study Group. Effectiveness of adjuvant chemotherapy in combination with tamoxifen for node-positive postmenopausal breast cancer patients. J Clin Oncol. 1997; 15:1385-1394. [PubMed: 9193330]

13. International Breast Cancer Study Group. Adjuvant chemotherapy followed by goserelin versus either modality alone for premenopausal lymph node-negative breast cancer: a randomized trial. J Natl Cancer Inst. 2003; 95:1833-1846. [PubMed: 14679153]

14. International Breast Cancer Study Group. Endocrine responsiveness and tailoring adjuvant therapy for postmenopausal lymph node-negative breast cancer: a randomized trial. J Natl Cancer Inst. 2002; 94:1054-1065. [PubMed: 12122096]

15. Whelan TJ, Clark RM, Levine MN, et al. The effect of delay in initiating radiotherapy postlumpectomy on local breast recurrence. Int J Radiat Oncol Biol Phys. 1996; 36:280.

16. Vujovic O, Perera F, Dar AR, et al. Does delay in breast irradiation following conservative breast surgery in node-negative breast cancer patients have an impact on risk of recurrence? Int $\mathrm{J}$ Radiat Oncol Biol Phys. 1998; 40:869-874. [PubMed: 9531372] 

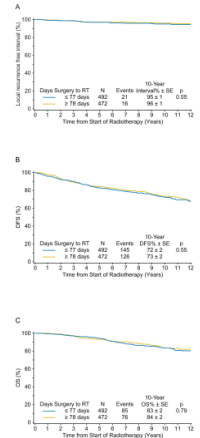

Figure 1.

Kaplan-Meier curves for time to local recurrence (A), disease-free survival (B) and overall survival (C) with time to event from start of radiotherapy for patients with radiotherapy within or after 77 days from surgery. 

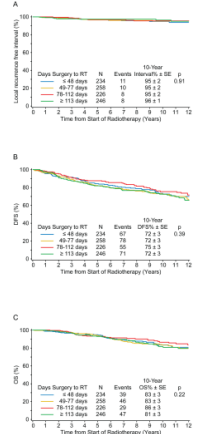

Figure 2.

Kaplan-Meier curves for time to local recurrence (A), disease-free survival (B) and overall survival (C) with time to event from start of radiotherapy as in quartiles ( $\leq 48$ days, $49-77$ days, 78-112 days and $\geq 113$ days). 
Table 1

Baseline Characteristics

\begin{tabular}{|c|c|c|c|}
\hline Characteristic & $\begin{array}{l}\text { RT within } 77 \text { Days } \\
\qquad(\mathrm{N}=492)\end{array}$ & $\begin{array}{l}\text { RT after } 77 \text { Days } \\
\quad(N=472)\end{array}$ & P-value* \\
\hline \multicolumn{4}{|c|}{ Mean $\pm S D$} \\
\hline \multirow[t]{2}{*}{ Age, years } & $55.6 \pm 8.9$ & $58.8 \pm 7.3$ & $<0.0001$ \\
\hline & Numbe & $(\%)$ & \\
\hline \multicolumn{4}{|c|}{ Menopausal status } \\
\hline Pre & $128(26)$ & $45(10)$ & $<0.0001$ \\
\hline Post & $364(74)$ & $427(90)$ & \\
\hline \multicolumn{4}{|l|}{ ER Status } \\
\hline Negative & $123(25)$ & 89 (19) & 0.042 \\
\hline Positive & $357(73)$ & $358(76)$ & \\
\hline Unknown & $12(2)$ & $25(5)$ & \\
\hline \multicolumn{4}{|l|}{ Tumor Grade } \\
\hline 1 & $89(18)$ & $86(18)$ & 0.94 \\
\hline 2 & $237(48)$ & $221(47)$ & \\
\hline 3 & $165(34)$ & $162(34)$ & \\
\hline Unknown & $1(0)$ & $3(1)$ & \\
\hline \multicolumn{4}{|c|}{ Number of Positive Lymph Nodes } \\
\hline 0 & 454 (92) & 441 (93) & 0.62 \\
\hline $1-3$ & $25(5)$ & $23(5)$ & \\
\hline $4+$ & $13(3)$ & $8(2)$ & \\
\hline \multicolumn{4}{|l|}{ Tumor Size } \\
\hline$\leq 2 \mathrm{~cm}$ & $371(75)$ & $366(78)$ & 0.35 \\
\hline$>2 \mathrm{~cm}$ & $118(24)$ & $100(21)$ & \\
\hline Unknown & $3(1)$ & $6(1)$ & \\
\hline \multicolumn{4}{|l|}{ Vessel Invasion } \\
\hline No & $378(77)$ & $364(77)$ & 0.098 \\
\hline Yes & 94 (19) & $67(14)$ & \\
\hline Unknown & $20(4)$ & $41(9)$ & \\
\hline
\end{tabular}

Unknowns for a given category are excluded from calculation of p-values. 
Table 2

Adjusted Proportional Hazards Regression Results Dividing at the Median

\begin{tabular}{lccc}
\hline RT Timing (from Surgery) & Hazard Ratio & 95\% CI & P-Value \\
\hline Local Recurrence & & & \\
$\leq 77$ Days & 0.94 & 0.47 to 1.87 & 0.86 \\
$\geq 78$ Days & 1.00 & & \\
Disease-Free Survival & & & \\
$\leq 77$ Days & 1.05 & 0.82 to 1.34 & 0.73 \\
$\geq 78$ Days & 1.00 & & \\
Overall Survival & & & \\
$\leq 77$ Days & 1.07 & 0.77 to 1.49 & 0.67 \\
$\geq 78$ Days & 1.00 & & \\
\hline
\end{tabular}

Table 2B. ER-positive cohort: Adjusted Proportional Hazards Regression Results Dividing at the Median

\begin{tabular}{lccc}
\hline RT Timing (from Surgery) & Hazard Ratio & 95\% CI & P-Value \\
\hline Local Recurrence & & & \\
$\leq 77$ Days & 0.84 & 0.38 to 1.89 & 0.68 \\
$\geq 78$ Days & 1.00 & & \\
Disease-Free Survival & & & \\
$\leq 77$ Days & 0.95 & 0.71 to 1.28 & \\
$\geq 78$ Days & 1.00 & & \\
Overall Survival & & & \\
$\leq 77$ Days & 1.16 & 0.77 to 1.74 & \\
$\geq 78$ Days & 1.00 & & \\
\hline
\end{tabular}


Table 3A

Adjusted Proportional Hazards Regression Results Based on Quartiles

\begin{tabular}{lccc}
\hline RT Timing (Days from Surgery) & Hazard Ratio & $\mathbf{9 5 \%}$ CI & P-Value \\
\hline Local Recurrence & & \multicolumn{2}{c}{ Overall Wald $P=0.99$} \\
$\leq 48$ & 0.90 & 0.34 to 2.37 & 0.83 \\
49 to 77 & 0.86 & 0.33 to 2.25 & 0.76 \\
78 to 112 & 0.89 & 0.33 to 2.41 & 0.81 \\
$\geq 113$ & 1.00 & & \\
Disease-Free Survival & & Overall Wald $P=0.12$ \\
$\leq 48$ & 0.78 & 0.55 to 1.10 & 0.16 \\
49 to 77 & 0.95 & 0.68 to 1.32 & 0.75 \\
78 to 112 & 0.68 & 0.47 to 0.97 & 0.03 \\
$\geq 113$ & 1.00 & & \\
Overall Survival & & Overall Wald $P=0.03$ \\
$\leq 48$ & 0.70 & 0.45 to 1.11 & 0.13 \\
49 to 77 & 0.86 & 0.56 to 1.31 & 0.47 \\
78 to 112 & 0.50 & 0.31 to 0.81 & 0.005 \\
$\geq 113$ & 1.00 & & \\
\hline
\end{tabular}

Table 3B. ER-positive cohort: Adjusted Proportional Hazards Regression Results Based on Quartiles

\begin{tabular}{lccc} 
RT Timing (Days from Surgery) & Hazard Ratio & 95\% CI & P-Value \\
\hline Local Recurrence & & \multicolumn{2}{c}{ Overall Wald $P=0.85$} \\
$\leq 48$ & 1.03 & 0.34 to 3.14 & 0.96 \\
49 to 77 & 0.67 & 0.21 to 2.17 & 0.50 \\
78 to 112 & 0.99 & 0.31 to 3.17 & 0.99 \\
$\geq 113$ & 1.00 & & \\
Disease-Free Survival & & Overall Wald $P=0.20$ \\
$\leq 48$ & 0.72 & 0.48 to 1.09 & 0.12 \\
49 to 77 & 0.82 & 0.55 to 1.22 & 0.32 \\
78 to 112 & 0.65 & 0.42 to 0.99 & 0.04 \\
$\geq 113$ & 1.00 & & \\
Overall Survival & & \multicolumn{1}{c}{ Overall Wald $P=0.05$} \\
$\leq 48$ & 0.76 & 0.44 to 1.32 & 0.33 \\
49 to 77 & 0.80 & 0.47 to 1.38 & 0.42 \\
78 to 112 & 0.42 & 0.23 to 0.78 & 0.005 \\
$\geq 113$ & 1.00 & & \\
\hline
\end{tabular}

